\title{
Introduction to the Minitrack "Simulation Modeling and Digital Twins for Decision Making in the Age of Industry 4.0"
}

\author{
Tobias Reggelin \\ Otto von Guericke \\ University Magdeburg \\ tobias.reggelin@ovgu.de
}

\author{
Stefan Galka \\ Ostbayerische Technische \\ Hochschule Regensburg \\ stefan.galka@oth- \\ regensburg.de
}

\author{
Dmitry Ivanov \\ Berlin School of \\ Economics and Law \\ divanov@hwr-berlin.de
}

\author{
Sebastian Lang \\ Fraunhofer Institute for \\ Factory Operation \\ and Automation IFF \\ Magdeburg \\ sebastian.lang@iff.fraunho \\ $\underline{\text { fer.de }}$
}

\begin{abstract}
The minitrack contains contributions with a focus on simulation modeling and digital twins and decision making in the context of Industry 4.0. The use of simulation models and digital twins is manifold, from planning to virtual commission and real-time operational decision support. Cyber-physical systems integrate the real world and the virtual world to enable decision making in the age of Industry 4.0.

For example, customer decisions and supply disruptions which directly influence manufacturing and logistics systems require immediate decisions. Thus, models must have the capability to support decision making in manufacturing as well as in internal and external logistics in real-time.

Methods include discrete-event simulation, discrete-rate simulation, hybrid simulation, system dynamics simulation, the combination of simulation modeling with machine learning or optimization heuristics, prescriptive analytics, and adaptive systems. Furthermore, this minitrack addresses simulation education and simulation models used for education and training in manufacturing and logistics.
\end{abstract}

\section{Deep reinforcement learning for supply chain synchronization}

Supply chain synchronization can help to prevent the bullwhip effect and significantly mitigate ripple effects caused by operational failures and other disturbances. The paper "Deep reinforcement learning for supply chain synchronization" by Jackson [1] shows how deep reinforcement learning agents based on the proximal policy optimization algorithm can synchronize inbound and outbound flows if end-toend visibility in a supply chain is provided. The proposed solution has the potential to perform adaptive control in complex supply chains. One advantage of the approach presented in the paper is that it is universally applicable and task-unspecific.

\section{Developing a Decision Support System for Integrated Decision-Making in Purchasing and Scheduling under Lead Time Uncertainty}

Decision making in supply chain management is complex due to the relationships between planning tasks from different planning phases and levels. Uncertainties such as unpredictable supplier delivery times and supply chain disruptions further complicate decision making. The paper of Rolf et al. [2] uses the case study of a company that assembles printed circuit boards to propose a concept for a decision support system. The global supply network is characterized by highly variable delivery times. As a result, the company maintains high inventory levels to prevent shortages. The decision support system considers the problems of purchasing and scheduling decisions in an integrated way. The prototype implementation of the purchasing algorithm uses a genetic algorithm that recommends reorder days and order quantities using a simulation model, and evaluates the risks of the solution by calculating the probability of stockouts.

\section{References}

[1] I. Jackson (2022). "Deep reinforcement learning for supply chain synchronization", Proceedings of the 55th Hawaii International Conference on System Sciences.

[2] B. Rolf, T. Reggelin, S. Lang, and S. Galka, "Developing a Decision Support System for Integrated DecisionMaking in Purchasing and Scheduling under Lead Time Uncertainty", Proceedings of the 55th Hawaii International Conference on System Sciences. 